\title{
Is intraoperative touch imprint cytology indicated in the surgical treatment of early breast cancers?
}

\author{
Z. Horváth ${ }^{\text {a }}$, A. Paszt ${ }^{\text {a }}$, Z. Simonka ${ }^{\text {a }}$, M. Látos ${ }^{\text {a }}$, V. Oláh ${ }^{\text {a }}$, \\ D. Nagyszegi ${ }^{\text {a }}$, L. Kaizer ${ }^{\text {b }, ~ Z . ~ F e j e s ~}{ }^{\text {b }}$, S. Hamar ${ }^{\text {b }}$, E. Csörgö ${ }^{b}$, \\ K. Ormándi ${ }^{\mathrm{c}}$, M. Lázár ${ }^{\mathrm{c}}$, G. Lázár ${ }^{\mathrm{a}, *}$ \\ ${ }^{a}$ Department of Surgery, University of Szeged, Albert Szent-Györgyi Clinical Center, Semmelweis u. 8., Szeged, \\ H-6720, Hungary \\ ${ }^{\mathrm{b}}$ Department of Pathology, University of Szeged, Albert Szent-Györgyi Clinical Center, Allomás u. 2., Szeged, \\ H-6720, Hungary \\ ${ }^{\mathrm{c}}$ Diagnoscan Hungary - Szeged, Semmelweis u. 6., Szeged, H-6725, Hungary
}

Accepted 10 January 2017

Available online 18 January 2017

\begin{abstract}
Introduction: Intraoperative touch imprint cytology (TIC) of the sentinel lymph node(s) (SLN(s)) in the treatment of breast cancer has significantly reduced the number of axillary block dissections (ABD) required during second surgeries. Based on recent studies, ABD was not considered necessary if the presence of tumor cells/micrometastasis was confirmed in the SLN(s) or in the case of macrometastases in a patient group meeting the inclusion criteria for the ACOSOG Z0011 study. Our aim was to determine the sensitivity and usefulness of TIC with regard to these results.

Methods: TICs of the SLN(s) were examined in 1168 patients operated on for breast cancer. The method was also analyzed retrospectively based on the guidelines for the Z0011 study. During TIC, new samples were cut every $250 \mu \mathrm{m}$; impression smears were evaluated after being stained with hematoxylin eosin.

Results: TIC confirmed metastasis in 202 cases (202/1168, 17.29\%). Metastasis was confirmed in SLN(s) in 149 additional cases during a final histological examination. The sensitivity of TIC was found to be $57.18 \%$, and its specificity was $99.63 \%$. An analysis was then performed except for cases that met the inclusion criteria for the Z0011 study and with metastasis smaller than 2 mm (micrometastasis/isolated tumor cells) considered to be positive during intraoperative cytology. The sensitivity of the method decreased to $34.23 \%$, while its specificity was still high at $99.76 \%$.

Conclusions: Based on the new guidelines for ABD, imprint cytology cannot be considered a beneficial and cost-effective intervention in the surgical treatment of early breast cancer.

(C) 2017 Elsevier Ltd, BASO The Association for Cancer Surgery, and the European Society of Surgical Oncology. All rights reserved.
\end{abstract}

\section{Introduction}

The introduction of sentinel lymph node biopsy (SLNB) in the treatment of early breast cancer 20 years ago significantly reduced the number of radical surgical interventions and the number of axillary block dissections (ABD). ${ }^{1,2}$ Intraoperative analysis of the sentinel lymph node(s) has been used increasingly, as surgeries performed in two sessions can be avoided with this method in most cases. ${ }^{1,3}$

\footnotetext{
* Corresponding author. Department of Surgery, University of Szeged, Albert Szent-Györgyi Clinical Center, Szőkefalvi-Nagy B. u. 6., Szeged, H-6720, Hungary. Fax: +36 62545701

E-mail addresses: horvath.zoltan@med.u-szeged.hu (Z. Horváth), paszt.attila@med.u-szeged.hu (A. Paszt), simonka.zsolt@med.u-szeged.hu (Z. Simonka), latos.melinda@med.u-szeged.hu (M. Látos),viktorolah2@gmail.com (V. Oláh), nagyszegi.dora@gmail.com (D. Nagyszegi), kaizer.laszlo@med.u-szeged.hu (L. Kaizer), fejes.zsuzsanna@med.u-szeged.hu (Z. Fejes), hamar.sandor@med.u-szeged.hu (S. Hamar), csorgo.erika@med.u-szeged.hu (E. Csörgő), ormikati@gmail.com (K. Ormándi), lazarmate@gmail.com (M. Lázár), lazar.gyorgy@med.u-szeged.hu (G. Lázár).
} 
Intraoperative histological examinations include imprint cytology, frozen section histology and a nucleic acid amplification study. The specificity and sensitivity of these examinations are similar. The sensitivity of the nucleic acid amplification study is $76.9-98.2 \%$, and that of frozen section histology and imprint cytology varies between 68.49 and $98.81 \%$. The specificity of all three methods is considered almost $100 \% .^{4-7}$

Surgical treatment of the axilla has changed significantly, particularly with regard to the indication for supplementary ABD. Based on the results of several prospective studies, ABD is not indicated for positive sentinel lymph nodes containing isolated tumor cells (ITC, $<0.2 \mathrm{~mm}$ ) and micrometastases $(<2 \mathrm{~mm})$, adjuvant therapy being sufficient. ${ }^{8-10}$ The results were also described in several international guidelines. ${ }^{9,11}$ Axillary block dissection is not necessarily required for patients with wide excision and sentinel lymph node biopsy for stage $T_{1}$ or $T_{2}$ breast cancer with sentinel lymph nodes containing no more than 1-2 macrometastases, according to the ACOSOG Z0011 study published in 2011 (neoadjuvant therapy is an exclusion criterion as well), as additional surgical treatment neither increases survival significantly nor reduces the incidence of axillary recurring tumors. ${ }^{8}$ In these cases, adjuvant systemic treatment and complete irradiation of the breast are sufficient. ${ }^{8,11-17}$ According to the ACOSOG Z0011 study criteria, a consensus conference held in St. Gallen in 2013, the Guidelines of the American Association of Oncology $(\mathrm{NCCN})$ and axillary block dissection may be omitted. $8,13,14$

According to several studies, ABD is an equivalent therapeutic alternative to targeted axillary radiation. ${ }^{15-17}$ Knowledge of treatment alternatives and participation in treatment selection represent a growing demand among patients. However, in the case of axillary block dissection performed for positive imprint cytology, patients cannot participate in the therapeutic decision.

In conclusion, the routine use of imprint cytology should be reconsidered. In our study, imprint cytology results were examined and analyzed retrospectively based on new national guidelines on the treatment of the axilla. Moreover, we analyzed the cost and time of breast surgery taking into account the necessity of imprint cytology.

sensitivity $=$ realpositivecases/realpositivecases + falsenegativecases

\section{Patients and methods}

Between 2008 and 2014, a total of 1673 patients underwent surgery in our institution for invasive breast cancer and other breast malignancies. In this period, 1168 patients who were suffering from consecutive early invasive breast carcinoma and whose preoperative axillary US + FNA did not show axillary lymph node metastasis were examined with imprint cytology of the SLN(s).

Sentinel lymph nodes were removed with the double tracer method published by Albertini in $1996 .{ }^{18}$ On the day before the surgery (but at least four hours before the surgery), human colloidal albumin was administered with isotope $(99 \mathrm{mTc})$ tracing under ultrasound or X-ray guidance near the lesion. A lymphoscintigraphic (static) examination was then performed to determine the projection of SLN(s) and lymphatic drainage. The patent blue dye was periareolarly. The SLN(s) were identified with a manual gamma camera.

The technique for touch imprint cytology (TIC) was as follows: the cut surface of the fresh sample prepared from a lymph node ( $250 \mu \mathrm{m}$ slices) was pressed on a slide, and then an impression smear was prepared. The resulting imprint cut surfaces were fixed in 95\% ethanol for 5-6 s, and the samples were evaluated after hematoxylin eosin staining. Every SLN underwent a standard histological examination later.

We allocated the sensitivity and specificity of TIC based on the intraoperative and final histological result. Based on the final histological examination of the sentinel lymph no$\mathrm{de}(\mathrm{s})$, we selected patients in whose cases performing an $\mathrm{ABD}$ is no longer justified according to the new international recommendations (ITC, micro- and macrometastases, which meet the criteria for ACOSOG Z0011) and thus there is no need for intraoperative lymph node analysis either. Based on these data, the imprint cytology results were re-evaluated and compared with non-modified data.

\section{Statistics}

We studied the sensitivity and specificity of imprint cytology with the scheme below:

specificity $=$ realnegativecases $/$ realnegativecases + falsepositivecases 
We used the SPSS (Statistical Package for the Social Sciences) programme for comparing the operating time. We analyzed the data two-sample T-test. The study has been approved by the local Ethics Committee.

\section{Results}

In our Department, TIC was performed for invasive breast tumor in 1168 cases during the period under examination. The average age of the patients was $58.63(25-88)$ years. TIC was positive in 202 cases in $17.29 \%(202 / 1168)$ of the cases. A total of 2101 lymph nodes were sent for imprint cytology, which is an average of 1.8 (1-6) lymph nodes per patient.

During a final histological examination of the samples, metastasis was found in 149 additional previously (intraoperatively) negative sentinel lymph nodes (false negative cases: 149/1168 [12.75\%]), and metastasis was not confirmed in three cases found to be positive with imprint cytology (false positive cases: $3 / 149$ [0.25\%]). The sensitivity of the imprint cytology was $57.18 \%$, with a specificity of $99.63 \%$ (Table 1 ).

A total of 202 axillary block dissections were performed in one session, and then 80 block dissections were performed on a separate occasion. In 64 cases, an ABD was not performed, as the patient had not given his consent for the intervention, the patient had chosen adjuvant chemotherapy, or the oncoteam had not recommended additional surgery due to the presence of micrometastasis.

At that point, patients meeting the inclusion criteria for the Z0011 study $(\mathrm{n}=117)$ and seven patients not meeting the criteria for Z0011 but with micrometastasis $(\mathrm{n}=7)$ were excluded from the positive cases by intraoperative examination (Tables 1 and 2), and then the sensitivity and specificity of the method were recalculated.

After screening, 78 positive (number of positive cases $=202$ minus number of patients meeting criteria

Table 1

Imprint cytology results (1 May 2008-31 Dec 2014).

\begin{tabular}{lll}
\hline & Total & $\%$ \\
\hline Number of patients & 1168 & 100 \\
Imprint cytology results & & \\
$\quad$ Intraoperative (positive) & 202 & 17.3 \\
$\quad$ Intraoperative (negative) & 966 & 82.7 \\
$\quad$ False negative & 149 & 12.76 \\
$\quad$ False positive & 3 & 0.25 \\
Number of positive final histological examinations & 348 & 29.79 \\
Number of negative final histological examinations & 820 & 70.21 \\
Type of surgery & & \\
$\quad$ Excision & 883 & 75.6 \\
$\quad$ Mastectomy & 285 & 24.4 \\
Average tumor size (mm) & 19.61 & \\
Average number of SLNBs & 1.8 & \\
ABDs & 284 & 24.32 \\
In one session & 204 & 17.47 \\
Supplementary & 80 & 6.85 \\
\hline
\end{tabular}

Table 2

Patients meeting the criteria for the Z0011 study and with a positive imprint cytology.

\begin{tabular}{lll}
\hline & Total & $\%$ \\
\hline $\begin{array}{l}\text { Number of patients } \\
\text { Imprint cytology results }\end{array}$ & 117 & 100 \\
$\quad$ Positive & 116 & 99.15 \\
$\quad$ Negative & 1 & 0.85 \\
$\quad$ False negative & 0 & \\
$\quad$ False positive & 1 & 0.85 \\
Number of final, positive histological examinations & 116 & \\
Type of surgery & & \\
$\quad$ Excision & 117 & 100 \\
$\quad$ Mastectomy & 0 & 0 \\
Average tumor size (mm) & 20.56286 \\
Average number of SLNBs & 1.97 & \\
ABDs & & \\
Performed in one session & 111 & 94.87 \\
Supplementary & 2 & 1.71 \\
Not performed & \\
\hline a Axillary block dissection was not performed in four cases when a pa- \\
tient made a request in advance to that effect or in the presence of \\
micrometastases.
\end{tabular}

for Z0011 [ $\mathrm{n}=117]$ and number of patients not meeting criteria for Z0011 but with micrometastasis [n $=7]$ ), two false positive, 149 false negative and 966 negative cases were found in the repeated imprint cytology group. Sensitivity fell to $34.23 \%$, and specificity remained $99.76 \%$ (Table 3).

As can be seen on Table 4, axillary block dissection was performed in 284 cases, but, based on the new guidelines, only 105 cases were indicated. 179 cases, that is, $15.32 \%$ of the cases, were performed unnecessarily.

As the results were processed, the average size of the metastases and the distribution of micro- and macrometastases in false negative and positive cases were examined as well (Table 5). In positive cases, the average size of the micrometastases was $1.52 \mathrm{~mm}$, while that of the macrometastases was $8.33 \mathrm{~mm}$. The average size of the micrometastases was $1.1 \mathrm{~mm}$, while that of the macrometastases was $4.79 \mathrm{~mm}$ in the false negative group. Therefore, it can be concluded that imprint cytology cannot be considered a sensitive intervention for surgeries on smaller metastases.

The costs of ABDs performed with imprint cytology and with supplementary imprint cytology were compared in financial terms. (The costs of SLNBs and ABDs performed during surgery are similar.) During imprint cytology, an average of five cut surfaces is prepared of the lymph node. A cut surface costs $€ 32.50$. The average price of histology performed during surgery is therefore $1.8^{*} € 32.50$, that is, €58.52. An ABD costs $€ 431.17$. Based on the results, the 1168 imprint cytologies cost $€ 68,359.23$, while the 76 supplementary ABDs cost $€ 32,768.49$. Therefore, the difference is $€ 35,586.73$. Patient hospital stay after ABD is longer (about one day) and more out-patient visits are required, thus further increasing the difference in expenses. This represents an extra charge of about $€ 100$ 
Table 3

Results modified in accordance with the criteria.

\begin{tabular}{lllll}
\hline & Imprint (all cases) & $\begin{array}{l}\text { Cases meeting } \\
\text { Z0011 criteria }\end{array}$ & $\begin{array}{l}\text { Cases not meeting Z0011 criteria but } \\
\text { involving micrometastasis }\end{array}$ & Results recalculated \\
\hline $\begin{array}{l}\text { Number of patients } \\
\quad \text { Imprint }\end{array}$ & 1168 & 117 & 7 & 1168 \\
$\quad \begin{array}{l}\text { Positive } \\
\text { Negative }\end{array}$ & 202 & 117 & 7 & 78 \\
Final histology & 966 & 0 & 0 & 966 \\
$\quad$ Positive & 348 & 116 & 7 & 225 \\
False positive & 3 & 1 & 0 & 2 \\
False negative & 149 & 0 & 0 & 149 \\
\hline
\end{tabular}

Table 4

Distribution of axillary block dissections.

\begin{tabular}{llll}
\hline & & Total number of ABDs \\
\hline Imprint & Supplementary ABDs & 80 & 284 \\
& ABDs performed in one session & 204 & 2 \\
Positive cases according to Z0011 criteria & Supplementary ABDs & 114 & 112 \\
& ABDs performed in one session & 56 & 58 \\
False negative cases according to Z0011 criteria & Supplementary ABDs & 2 & 0 \\
& ABDs performed in one session & 7 & 7 \\
Cases not meeting Z0011 criteria but involving micrometastasis & Supplementary ABDs & 22 & 105 \\
& ABD performed in one session & 83 & \\
\hline
\end{tabular}

per patient (based on Hungarian expenses). We have examined the average duration of breast surgery with and without imprint cytology. Surgeries were longer by approximately $1.6-5$ min when cytology was used, and the difference was non-significant (data not shown).

\section{Discussion}

The introduction of SLNB has been a milestone in the treatment of early breast cancer, as it has significantly reduced the number of axillary block dissections and similarly improved staging and oncology/complex treatment of breast cancers. Simultaneously, intraoperative examination of the sentinel lymph node(s) has been introduced, and, as a result, the number of surgeries performed in two stages and, consequently, both the burden on the patients and surgical costs have decreased.

According to recent studies and guidelines, in cases meeting certain criteria, such as isolated tumor cells, sentinel lymph nodes with micrometastasis and no more than two macrometastases, ABD may be omitted, as it does not increase the risk of overall mortality and locally recurring cancer. ${ }^{8,9,11}$ Similarly, the indication for ABD is reduced by clinical studies supporting the fact that axillary radiation is an alternative treatment option to surgery, but it is associated with lower morbidity. ${ }^{15-17}$ Axillary radiotherapy does not pose an increased risk to survival and locally recurring cancer, and the incidence of lymphedema, which is primarily responsible for morbidity, is significantly decreased as well. Another important factor is the fact that in the case of axillary block dissection performed for positive imprint cytology, the patient is unable to participate in the therapeutic decision and choose from among therapeutic options.

These factors suggest a reconsideration of the routine use of imprint cytology. Based on our results, the sensitivity of imprint cytology is $57.18 \%$, its specificity is $99.63 \%$, and these values are consistent with international data. (The

Table 5

Lymph node metastases in false negative and false positive cases.

\begin{tabular}{|c|c|c|c|c|c|}
\hline & $\begin{array}{l}\text { Sizes of } \\
\text { metastases }(\mathrm{mm})\end{array}$ & $\begin{array}{l}\text { Number of } \\
\text { metastases }\end{array}$ & $\begin{array}{l}\text { Distribution in \% of } \\
\text { all metastases }\end{array}$ & $\begin{array}{l}\text { Distribution in \% of } \\
\text { all micro-metastases }\end{array}$ & $\begin{array}{l}\text { Distribution in \% of } \\
\text { all macro-metastases }\end{array}$ \\
\hline \multicolumn{6}{|l|}{ Positive cases } \\
\hline Micrometastases & 1.52 & 13 & 3.73 & 15.85 & \\
\hline Macrometastases & 8.33 & 187 & 53.58 & & 70.3 \\
\hline \multicolumn{6}{|l|}{ False negative cases } \\
\hline Micrometastases & 1.10 & 69 & 19.77 & 84.15 & \\
\hline Macrometastases & 4.79 & 80 & 22.92 & & 29.7 \\
\hline
\end{tabular}


sensitivity of imprint cytology varies between 68.49 and $98.81 \%$, with a specificity of approximately $100 \%){ }^{5,19-21}$ With regard to metastasis sizes and distribution, imprint cytology is less suited to detecting metastases of $2 \mathrm{~mm}$ or smaller, with $84.15 \%$ of micrometastases remaining undetected, but $70.3 \%$ of macrometastases can be detected with this method.

However, according to the latest guidelines, the sensitivity of imprint cytology (based on cases with therapeutic consequences) is only $34.23 \%$ (with unchanged specificity). This sensitivity value is so low that the usability of this method is questionable. ABD was only indicated in $9 \%$ of the patients in the period under examination (105/ 1168). However, based on the new guidelines, supplementary lymph node dissection was performed "unnecessarily" in $15.32 \%$ of the patients $(179 / 1168)$.

It is not insignificant that imprint cytology increased surgical costs and the duration of surgery unnecessarily in a large percentage of the patients (91\%). Imprint cytology and surgical costs are significantly higher in Western Europe and the United States; differences in costs (cost effectiveness) are therefore more significant in those regions (due to the operational cost of the surgery and the surgeon's fee, these interventions cost at least $€ 32-100$ per surgery, totaling $€ 37,376-116,800$ for 1168 patients. ${ }^{22}$ )

The price of imprint cytology of a lymph node in the United States in 2010 was (the dollar equivalent of) $€ 57 .^{23}$ Based on this data, in our case, the total cost would be $€ 119,832$. If we use the latest intraoperative histological diagnostic method, nucleic acid amplification, it costs $€ 172.50$ to examine a lymph node, based on data from a French survey. ${ }^{24}$ The cost for the total patient population was $€ 362,664$. Intraoperative histological examinations are less effective $e^{4,6}$ and significantly increase treatment costs for patients operated on for early breast cancer.

Our study shows that axillary block dissection is only necessary in the treatment of the axillary region in an increasingly smaller group of patients (9\%), and this percentage will further decrease with more extensive use of alternative axillary radiotherapy.

Based on our results, imprint cytology of the sentinel lymph node(s) in patients operated on for malignant breast cancer has no confirmed benefits based on the current guidelines, and its routine use is not indicated. According to the latest international guidelines, intraoperative examination of the sentinel lymph node(s) may be indicated in the case of mastectomy (when postoperative radiotherapy is not planned) and after neoadjuvant therapy, as ABD is still indicated in these cases.

\section{Conflict of interest statement}

The authors have no conflict of interest or financial disclosure.

\section{References}

1. Giuliano AE, Kirgan DM, Guenther JM, et al. Lymphatic mapping and sentinel lymphadenectomy for breast cancer. Ann Surg 1994;220:391401.

2. Giuliano AE, Dale PS, Turner RR, et al. Improved axillary staging of breast cancer with sentinel lymphadenectomy. Ann Surg 1995;222: 394-401.

3. Krag DN, Weaver DL, Alex JC, Fairbank JT. Surgical resection and radiolocalization of the sentinel lymph node in breast cancer using a gamma probe. Surg Oncol 1993;2:335-40.

4. Le Frère-Belda MA, Bats AS, Gillaizeau F, et al. Diagnostic performance of one-step nucleic acid amplification for intraoperative sentinel node metastasis detection in breast cancer patients. Int J Cancer 2012;130:2377-86.

5. Safai A, Razeghi A, Monabati A, Azarpira N, Talei A. Comparing touch imprint cytology, frozen section analysis, and cytokeratin immunostaining for intraoperative evaluation of axillary sentinel lymph nodes in breast cancer. Indian J Pathol Microbiol 2012;55: 183-6.

6. Cserni G. Intraoperative analysis of sentinel lymph nodes in breast cancer by one-step nucleic acid amplification. J Clin Pathol 2012; 65:193-9.

7. Pathmanathan N, Renthawa J, French JR, et al. Intraoperative sentinel lymph node assessment in breast cancer: a comparison of rapid diagnostic method based on CK19 mRNA expression and imprint cytology. ANZ J Surg 2014;84:730-4.

8. Giuliano AE, McCall L, Beitsch P, et al. Locoregional recurrence after sentinel lymph node dissection with or without axillary dissection in patients with sentinel lymph node metastases: the American College of Surgeons Oncology Group Z0011 randomized trial. Ann Surg 2010;252:426-32.

9. Goldhirsch A, Wood WC, Coates AS, et al. Strategies for subtypes-dealing with the diversity of breast cancer: highlights of the St. Gallen International Expert Consensus on the Primary Therapy of Early Breast Cancer 2011. Ann Oncol 2011;22:1736-47.

10. Solá M, Alberro JA, Fraile M, et al. Complete axillary lymph node dissection versus clinical follow-up in breast cancer patients with sentinel node micrometastasis: final results from the multicenter clinical trial AATRM 048/13/2000. Ann Surg Oncol 2013;20:120-7.

11. AGO, DGS, SGS, ÖGS, Panelists, Executive Board Members. German, Austrian and Swiss consensus conference on the diagnosis and local treatment of the axilla in breast cancer. Eur $J$ Cancer 2013;49:2277-83.

12. Galimberti V, Cole BF, Zurrida S, et al. Axillary dissection versus no axillary dissection in patients with sentinel-node micrometastases (IBCSG 23-01): a phase 3 randomised controlled trial. Lancet Oncol 2013;14:297-305.

13. Goldhirsch A, Winer EP, Coates AS, et al. Personalizing the treatment of women with early breast cancer: highlights of the St Gallen International Expert Consensus on the Primary Therapy of Early Breast Cancer 2013. Ann Oncol 2013;24:2206-23.

14. National Comprehensive Cancer Network. Clinical practice guidelines in oncology - breast cancer version 1.2016. [cited 2016 May10]. Available from: URL: http://www.nccn.org/professionals/physician_ gls/pdf/breast.pdf.

15. Rutgers EJ, Donker M, Straver ME, et al. Radiotherapy or surgery of the axilla after a positive sentinel node in breast cancer patients: final analysis of the EORTC AMAROS trial (10981/22023). J Clin Oncol 2013;31(Suppl). abstr LBA1001.

16. Donker M, van Tienhoven G, Straver ME, et al. Radiotherapy or surgery of the axilla after a positive sentinel node in breast cancer (EORTC 10981-22023 AMAROS): a randomised, multicentre, openlabel, phase 3 non-inferiority trial. Lancet Oncol 2014;15:1303-10.

17. Sávolt A, Polgár Cs, Musonda P, et al. Does the result of completion axillary lymph node dissection influence the recommendation for 
adjuvant treatment in sentinel lymph node-positive patients? Clin Breast Cancer 2013;13:364-70.

18. Albertini JJ, Cruse CW, Rapaport D, et al. Intraoperative radiolympho-scintigraphy improves sentinel lymph node identification for patients with melanoma. Ann Surg 1996;223:217-24.

19. Lumachi F, Marino F, Zanella S, Chiara GB, Basso SM. Touch imprint cytology and frozen-section analysis for intraoperative evaluation of sentinel nodes in early breast cancer. Anticancer Res 2012;32:3523-6.

20. Francz M, Egervari K, Szollosi Z. Intraoperative evaluation of sentinel lymph nodes in breast cancer: comparison of frozen sections, imprint cytology and immunocytochemistry. Cytopathology 2011;22:36-42.

21. Clarke D, Leung E, Chachlani N, et al. Intraoperative assessment of sentinel node using imprint cytology. World J Surg 2010;34:55-61.
22. Genta F, Zanon E, Camanni M, et al. Cost/accuracy ratio analysis in breast cancer patients undergoing ultrasound-guided fine-needle aspiration cytology, sentinel node biopsy, and frozen section of node. World J Surg 2007;31:1155-63.

23. Kamiński JP, Case D, Howard-McNatt M, Geisinger KR, Levine EA. Sentinel lymph node intraoperative imprint cytology in patients with breast cancer-costly or cost effective? Ann Surg Oncol 2010;17: 2920-5.

24. Raia-Barjat T, Trombert B, Khaddage A, et al. OSNA (one-step nucleic acid amplification) sentinel lymph node intraoperative molecular analysis in breast cancer: a cost-benefit analysis. Med Oncol 2014;31: 322. 